\title{
A Correlative Multi-Spectroscopy and Docking Study for the Modeling of Drug (Luteolin and Quercetin) Binding to Bovine Serum Albumin- A Tool for the Determination of Binding Characteristics to Receptor Proteins
}

\author{
Suresh Palamadai Krishnan*, Kaustubh Sunil Hiray, Siddhant Vyas \\ Department of Biomedical Sciences, School of Biosciences and Technology, VIT, Vellore, Tamil Nadu, INDIA.
}

\begin{abstract}
The in vitro in silico experimental flow (multi- spectroscopy and docking) demonstrated the binding of Luteolin and Quercetin separately with Bovine Serum Albumin. For the first time, we are reporting the relative UV-visible spectroscopy-based hypsochromic shifts for both luteolin $(3 \mathrm{~nm})$ and quercetin $(4.1 \mathrm{~nm})$ respectively. The drug-induced conformational change may lead to the possible shift in the tryptophan residue to a more hydrophobic environment. Our demonstration of an increased static quenching of the endogenous fluorophore in BSA validated the UV-visible spectroscopy data. However, detailed experiments will further delineate the possible relative contribution of dynamic quenching processes. The strong binding (binding constant values $-10^{5} \mathrm{~L} / \mathrm{mol}$ ) and the number of binding sites ( 1 for luteolin and quercetin) is consistent with published findings. Under our defined conditions, the hitherto unreported non-cooperative binding was demonstrated, based on the Hill's coefficient. Thermodynamic data qualitatively validated hydrophobicity (a positive entropy change $\Delta \mathrm{S}_{0}$ ); hydrogen bonding (a negative $\Delta \mathrm{H}_{0}$ ) and electrostatic interactions (a negative $\Delta \mathrm{H}_{0}$ and a positive $\Delta \mathrm{S}_{0}$ ). For the first time, the Infra-Red Spectroscopy (FT-IR) data showed ground state complex formation of the molecules with the model protein and may serve to corroborate our fluorescence (static quenching) data. Hydrogen bonds and hydrophobic interactions for both molecules (Ligplot Analysis) provide corroborative evidence for the molecular spectroscopy and thermodynamic data. This hitherto unreported, unique, combinatorial in vitro (multispectroscopy and thermodynamic measurements) in silico (docking and Ligplot-based analysis) experimental flow (specifically for luteolin and quercetin) provides a basis for extending such binding studies for novel receptors and/or ligands.
\end{abstract}

Key words: Bovine Serum Albumin (BSA), Luteolin/Quercetin, Fluorescence spectroscopy, FT-IR spectroscopy, AUTODOCK/LIGPLOT.

\section{INTRODUCTION}

Screening of molecules for their chemo-preventive and/or chemotherapeutic potential has been performed globally (individually or High-Throughput Screening) for quite some time now. In this regard, binding characteristic studies using model hydrophobic proteins have been determined to model distribution (pharmacokinetic variable affecting storage and transport) and certain pharmacodynamic characteristics (affecting bioavailability and anti-oxidant potential). With the similarity being 76\%, Bovine Serum albumin (BSA) may be a model counterpart of the human protein. BSA has 3 domains (I, II and III) homologous to each other with each of the domains
Submission Date: 05-11-2017; Revision Date: 05-12-2017; Accepted Date: 12-12-2017

DOI: 10.5530/ijper.52.3.57 Correspondence: Suresh $\boldsymbol{P}$ K,

Department of Biomedical Sciences, School of Biosciences and Technology, VIT University, Vellore 632014, Tamil Nadu, INDIA. Phone: 0416-2202474 E-mail: p.k.suresh@vit.ac.in

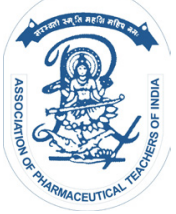

www.ijper.org 
forming two subdomains (for e.g., $1 \mathrm{~A}$ and $1 \mathrm{~B}$ ). ${ }^{1}$ These domains are divided into 9 loops with 17 disulfide bonds. There are three loops in each of the domains and they form a triplet (large-small-large loop). The protein is largely $\alpha$-helical with no $\beta$ sheets (the remainder of the protein is largely turns or extended and flexible regions between subdomains). Specifically, the binding behaviour of BSA can be monitored based on the properties of the endogenous, fluorescent, two tryptophan residues (Trp-134 is on the outside $-1^{\text {st }}$ domain and Trp-212 is in the interior, hydrophobic binding pocket $-2^{\text {nd }}$ domain), following exposure to pharmacologically relevant biomolecules (luteolin or quercetin) that have quenching properties (can also be used as experimental probes for proteins with endogenous fluorophores in them). ${ }^{2,3}$ Since bovine serum albumin is readily available and is relatively inexpensive, this protein continues to be the model of choice for the homologous Human Serum Albumin. This protein has been shown to be evolutionarily conserved and is involved in the transport of drugs and xenobiotics. Hence, binding behaviour to such a molecule may be a predictor of the distribution determinants of model drugs in humans, despite some reports questioning its validity especially with respect to it being able to accurately model HSA. ${ }^{4}$ BSA has been used to predict binding behaviour of drugs, since both the proteins (BSA and HSA) have a similar primary structure and folding pattern with HSA have one tryptophan residue unlike BSA. Further, analytical techniques can be used to better understand certain Structure-Activity Relationship (SAR)-based correlative variations in some of the pharmacodynamic variables. This paper discusses the Characteristic shifts in the UV-visible spectra demonstrating the role of the possible binding-induced changes in the interactions with tyrosine and tryptophan. The comparative fluorescence spectra was obtained under optimized conditions (data not shown) and the quenching phenomena was demonstrated. Further, a comparison has been made with regards to the number and site of binding (cooperativity and the Hill coefficient) under optimized conditions. We have shown an FT-IR-based comparison of the interactions of luteolin and quercetin with BSA. Comparison has been made with regards to the thermodynamic aspects. Finally, AUTODOCKbased binding studies were done and LIGPLOT-based visualization of the docked complexes was performed. Our experimental design involves for the first time, the demonstration of the relative hypsochromic UV-visible spectroscopy-based shifts of $3 \mathrm{~nm}$ and $4.1 \mathrm{~nm}$ for Luteolin and Quercetin respectively. We have also validated the static nature of the drug-based fluorescence quenching of the flurophore in the BSA. Further, we are, for the first time reporting on the non-cooperative nature of the binding as per the Hill's coefficient values. To the best of our knowledge, this is the first report of its kind wherein FT-IR spectra has been discussed for BSA interacting with luteolin and quercetin separately. To the best of our knowledge, this experimental, combinatorial in vitro in silico flow has not hitherto been specifically reported for the comparative study involving luteolin and quercetin. Also, this flow can be used by academicians as a teaching tool that can be extended for research purposes for novel receptors and novel ligands. This work will pave the way for validation of in vitro/spectroscopy studies with human serum albumin for these and other compounds in the flavonoid class. This approach will thereby also aid in the evaluation of the predictability of the nature and site of interactions for all categories of natural compounds (existing and novel), not necessarily restricted to the flavonoid class of compounds.

\section{MATERIALS}

Bovine Serum Albumin, Fraction V (BSA, fatty acidfree $>99 \%$ ) was procured from HiMedia, Mumbai, India. Luteolin and Quercetin were procured from Sigma-Aldrich, India.

The buffer system used was Phosphate Buffered Saline (PBS, 0.02M), pH 7.4 and was filtered using a $0.4 \mu \mathrm{m}$ filter paper. Double-distilled water was used for all the experiments. Quercetin $\left(3.3 \times 10^{-4} \mathrm{M}\right)$ and Luteolin stock solutions $(100 \mu \mathrm{M})$ were prepared in ethanol $(>99 \%)$ and then diluted with PBS to obtain the desired concentrations.

\section{Apparatus}

The UV-VIS absorbance data was measured using the Varian Cary 50 UV-VIS Spectrophotometer and results were recorded using Varian UV Scan Application. All the fluorescent spectral analysis was carried out using the F-7000 FL Spectrophotometer (Hitachi, Japan). This instrument was equipped with a $150 \mathrm{~W}$ xenon lamp source and a $1.0 \mathrm{~cm}$ cell. The excitation wavelength was $280 \mathrm{~nm}$. The emission wavelength band was obtained between $300 \mathrm{~nm}-400 \mathrm{~nm}$. The excitation and emission slit width was $5.0 \mathrm{~nm}$ throughout the experiments. The results were recorded with the help of standard software- FL Solutions program. The FTIR (Fourier Transform Infra-Red) Spectroscopy was carried out on Shimadzu IRAffinity-1S, FTIR Spectrophotometer. The device is equipped with a Michelson interferometer and also has a dynamic alignment system. The ATR mode was used for the experiment and the data processing 
and analysis was carried out using the Lab Solutions IR software.

\section{METHODS}

All the experiments were performed at least twice. Representative images for the different methods have been included in the results section.

\section{UV-Vis Spectroscopy}

The concentration of Luteolin and Quercetin was respectively varied, whereas BSA concentration was kept constant $\left(1.51 \times 10^{-5} \mathrm{M}\right)$. The UV-Vis spectral scan was done to identify the $\lambda_{\text {max }}$ of the respective molecules (luteolin, quercetin, BSA -scans not shown). The samples were in a $1 \mathrm{~cm}$ optical path quartz cuvette. Quercetin samples in the range $3.3 \mu \mathrm{M}$ to $33 \mu \mathrm{M}$ were prepared in ethanol and diluted accordingly with $0.02 \mathrm{M}$ PBS, $\mathrm{pH}$ 7.4. ${ }^{1,4}$ The varying concentrations of Quercetin were then complexed with BSA $\left(1.51 \times 10^{-5} \mathrm{M}\right)$ and incubated at $298 \mathrm{~K}\left(25^{\circ} \mathrm{C}\right)$ and $310 \mathrm{~K}\left(37^{\circ} \mathrm{C}\right)$ for $45 \mathrm{~min}$ (optimization of the time was done $-15 \mathrm{~min}$ and $45 \mathrm{~min}$ respectively-data not shown). For a mole to mole comparison, Luteolin samples were also prepared in the same concentration range and in the same manner. The chemical was complexed with BSA $\left(1.51 \times 10^{-5} \mathrm{M}\right)$ and incubated under identical conditions of temperature, $\mathrm{pH}$ and time as mentioned earlier.

\section{Fluorescence Spectroscopy}

The Fluorescence Spectroscopy was carried out under similar reaction conditions as was done for the UV-Vis measurements. As before, BSA levels were kept constant $1.51 \times 10^{-5} \mathrm{M}(1 \mathrm{mg} / \mathrm{ml})$ while the concentration of luteolin and quercetin (fluorescence quenchers) were varied. The excitation $\lambda_{\text {max }}$ was set at $280 \mathrm{~nm}$ (based on the UV-visible data). Subsequently, the Fluorescence spectral scan was recorded between $200 \mathrm{~nm}$ to $800 \mathrm{~nm}$ to determine the emission wavelength. Two different temperatures $\left(298 \mathrm{~K}\left(25^{\circ} \mathrm{C}\right)\right.$ and $\left.310 \mathrm{~K}\left(37^{\circ} \mathrm{C}\right)\right)$ were use to determine the Fluorescence intensity of BSA in the presence and absence of the quenchers (Quercetin and Luteolin) as was the case for the UV-visible spectroscopy measurements. ${ }^{5,6}$ Sample preparation methodology was the same as was done for the generation of UV-visible data for both luteolin and quercetin respectively. Apart from the temperature conditions mentioned above, the other reaction conditions were also kept constant at the optimized $\mathrm{pH}$ (7.4) and incubation time (45 min).

\section{FTIR Spectroscopy}

As in the case of UV-visible and fluorescence spectroscopy, the BSA concentration was maintained at $1.51 \times 10^{-5} \mathrm{M}$
$(1 \mathrm{mg} / \mathrm{ml})$ while varying the aforesaid quencher concentrations. FTIR Spectroscopy was carried out for BSA alone (concentration $1.51 \times 10^{-5} \mathrm{M}$ at $298 \mathrm{~K}\left(25^{\circ} \mathrm{C}\right)$ and $310 \mathrm{~K}\left(37^{\circ} \mathrm{C}\right)$. Further, FT-IR measurements were also made after luteolin and quercetin $(33 \mu \mathrm{M}$ of both the flavonoids) were complexed to BSA individually at a temperature of $298 \mathrm{~K}\left(25^{\circ} \mathrm{C}\right)$ and $310 \mathrm{~K}\left(37^{\circ} \mathrm{C}\right)$. The flavonoid concentrations were chosen based on the results obtained from the fluorescence data. The IR spectra was obatined within a wavenumber range of $500 \mathrm{~cm}^{-1}$ to $4000 \mathrm{~cm}^{-1}$.

\section{Molecular Docking}

Molecular docking was done to validate the nature of the binding sites on BSA as well as the binding energy of the drug-protein complex. Further, LIGPLOT was used to identify and visualize the chemical nature of binding. The crystalline structure of BSA was obtained from PDB (PDB ID 4F5S), with a resolution of 2.47 Ä. Luteolin and Quercetin structures were obtained from PDB Ligand Expo (LU2 and QUE). The water molecules were excluded and hydrogen atoms were incorporated to this protein structure. Molecular docking was carried out using the docking program AutoDock 4.2. For identifying the binding domains of the drugs on the protein, BSA was docked with Luteolin and Quercetin separately. The grid box size of $126 \AA \times 126 \AA \times 126 \AA$ with a grid spacing of $1.2 \AA$ was constructed, and the grid maps were calculated using AutoGrid. Thirty (30) docking runs were performed for each drug-protein complex. Docking calculations were done using the Lamarckian Genetic Algorithm. The best-docked model (the one with the lowest binding energy) was then selected to represent the most favourable binding mode. The output of this docking is visualized using LIGPLOT. A 76\% homology has been observed between BSA and HSA with conserved repeating patterns of disulphides. The HSA structure was obtained from PDB (PDB ID 1AO6). A similar docking was done to explore the binding sites of the drugs on HSA. This approach could provide some assistance in comparing the results of BSA with the in silico HSA docking data. This in vitro in silico experimental flow would also aid in evaluating the utility of model protein binding data (as those of ours) as a predictive tool for the nature and site of binding of luteolin and quercetin-like molecules to similar hydrophobic proteins in humans.

\section{RESULTS}

UV-visible spectroscopy showed characteristic spectra for BSA, luteolin (band I and Band II) and quercetin respectively (data not shown), thereby validating the 
quality of the respective chemicals used in this study. The first band $(300 \mathrm{~nm}-400 \mathrm{~nm})$ is associated with cinnamoyl system, whereas the second band $(240 \mathrm{~nm}$ $280 \mathrm{~nm})$ is associated with the benzoyl system. ${ }^{7}$ In other words, the absorption of the $\mathrm{B}$ ring was due to $\mathrm{B}$ and $\mathrm{I}$ in the $320-385 \mathrm{~nm}$ range, while A ring absorption would be responsible for band II (250-285 nm). UV-visible spectra of the complexed chemicals (quercetin and luteolin respectively with BSA) showed an increase in the UV absorbance intensity of BSA with increasing concentration of both flavonoids. A characteristic hypsochromic shift (Blue shift) of $4.1 \mathrm{~nm}$ and $3 \mathrm{~nm}$ respectively was observed (Figure 1 and Figure 2).

Fluorescence emission spectroscopy data (slit width $5 \mathrm{~nm}$, excitation wavelength $280 \mathrm{~nm}$ ) was generated under identical reaction conditions (temperature $25^{\circ} \mathrm{C}$ and $37^{\circ} \mathrm{C}$, incubation time- $45 \mathrm{~min}, \mathrm{pH} 7.4$-similar to those maintained for the UV-visible spectroscopy data) with the BSA concentration kept constant with varying levels of luteolin and quercetin $(3.3 \mu \mathrm{M}$ to $33 \mu \mathrm{M})$ respectively. There wasadropin the fluorescenceintensity of BSA as the concentration of Luteolin was increased. A hypsochromic shift (Blue shift) is observed (Figure 3).

The classical Stern-Volmer equation (please refer to equation 1 below) was applied to demonstrate the quenching phenomenon for both luteolin and quercetin. Since the values of $\mathrm{k}_{\mathrm{Q}}$ were higher than the limiting rate constant of BSA i.e. $2 \times 10^{10} \mathrm{~L} / \mathrm{mol} . \mathrm{s}$, the mechanism involves, at least in part, a static quenching mechanism. ${ }^{8,9}$ The $\mathrm{K}_{\mathrm{SV}}$ values decreased with an increase in temperature. (Table $1 \mathrm{a}$ and Table $1 \mathrm{~b}$ )

The equation is represented as: where

$$
\mathrm{F}_{\mathrm{o}} / \mathrm{F}=1+\mathrm{kQ} \tau_{0}[\mathrm{Q}]=1+\mathrm{Ksv}[\mathrm{Q}] \quad \text { - Equation } 1
$$

$F_{0}-$ Fluorescence intensity without the quencher

F - Fluorescence intensity with the quencher

Ksv - Stern-Volmer quenching constant

[Q] - Quencher concentration

$\tau_{0}$ - Average fluorescence lifetime of the biomolecule without the quencher

$\mathrm{kQ}$ - Quenching rate constant

In order to obtain an insight into the nature of binding, binding constant $(\mathrm{K})$ values and the number of binding sites (n) per albumin molecule were calculated.

$\log \left(\left(\mathrm{F}_{0}-\mathrm{F}\right) / \mathrm{F}\right)=\log \mathrm{K}+\mathrm{n} \log [\mathrm{Q}] \quad-$ Equation 2 (double logarithmic equation) where,

$\mathrm{F}_{0}$ - Fluorescence intensity without the quencher

F- Fluorescence intensity with the quencher

[Q]- Quencher concentration

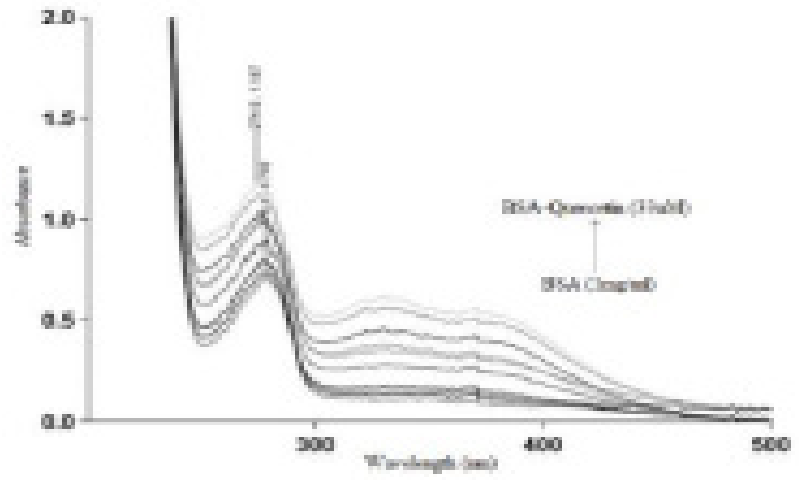

Figure 1: Ultra-Violet/Visible spectra of Bovine Serum Albumin complexed with Quercetin; temperature 37 degree C, incubation time- $\mathbf{4 5} \mathrm{min}, \mathrm{pH}$ 7.4. Observation: There was an increase in the UV absorbance intensity of Bovine Serum Albumin as the concentration of Quercetin was increased. A hypsochromic shift (Blue shift) of $4.1 \mathrm{~nm}$ was observed.

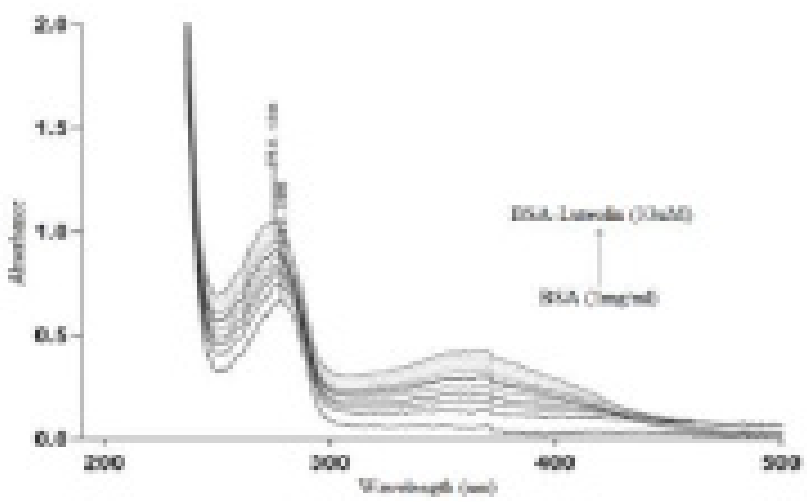

Figure 2: Ultra-Violet/Visible spectra of Bovine Serum Albumin complexed with Luteolin; temperature 37 degree $\mathrm{C}$, incubation time- $45 \mathrm{~min}, \mathrm{pH}$ 7.4. As the concentration of Luteolin was increased, there was an increase in the UV absorbance intensity of Bovine Serum Albumin. A hypsochromic shift (Blue shift) of $3.0 \mathrm{~nm}$ was observed.

The values of $\mathrm{K}$ were of the order of $10^{5} \mathrm{~L} / \mathrm{mol}$ or greater, which indicated that there was good binding between flavonoids (Luteolin and Quercetin) and BSA. The values for $\mathrm{n}$ obtained are approximately equal to 1 . This suggested that there was only one class of binding site for Luteolin and Quercetin on BSA respectively. It also shows that $\mathrm{K}$ and $\mathrm{n}$ decreased with an increase in temperature, which may indicate that a complex was formed subsequent to the binding (Table $2 \mathrm{a}$ and Table $2 \mathrm{~b})$. The following interactions were considered to be important in the binding reactions. They were hydrogen bonding, van der waals forces, hydrophobic interactions and electrostatic interactions. Studies using thermodynamic parameters can provide us with information pertaining to the major forces that can contribute 


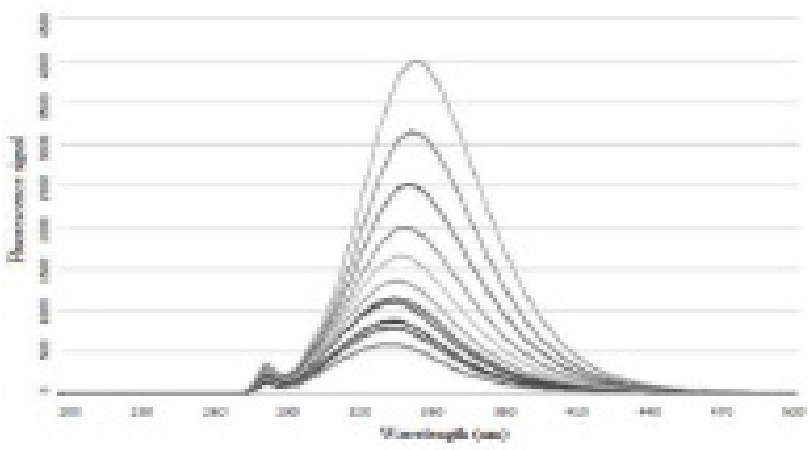

Figure 3 : Fluorescence emission spectra of Bovine Serum Albumin complexed with Luteolin ranging from $3.3 \mu \mathrm{M}$ to $33 \mu \mathrm{M}$ (slit width $5 \mathrm{~nm}$, excitation wavelength $280 \mathrm{~nm}$, temperature 25 degree $\mathrm{C}$, incubation time- $45 \mathrm{~min}, \mathrm{pH}$ 7.4). There was a drop in the fluorescence intensity of Bovine Serum Albumin as the concentration of Luteolin was increased. A hypsochromic shift (Blue shift) is observed. A decrease in the fluorescence intensity of Bovine Serum Albumin was also observed as the concentration of luteolin became higher. A similar hypsochromic shift (Blue shift) is observed. to protein stability following complexation with certain biomolecules.

The signs and magnitudes of the change in enthalpy $(\Delta \mathrm{H})$ and entropy $(\Delta \mathrm{S})$ can possibly account for the main forces contributing to protein stability.

$(\Delta \mathrm{H})$ and $(\Delta \mathrm{S})$ can be calculated from the Van't Hoff equation, provided the change in enthalpy does not change significantly at the temperature range tested.

The Van't Hoff equation is as follows:

$$
\ln \mathrm{K}=-\Delta \mathrm{H} / \mathrm{RT}+\Delta \mathrm{S} / \mathrm{R} \quad-\text { Equation } 3
$$

$\mathrm{K}$ is the binding constant at the corresponding temperature and $\mathrm{R}$ is the gas constant. The temperatures used were $25^{\circ} \mathrm{C}$ and $37^{\circ} \mathrm{C}$. The slope of the Van't Hoff relationship is used to calculate $\Delta \mathrm{H}$.

The Gibbs free energy change $(\Delta G)$ is estimated from the following relationship:

$$
\Delta \mathrm{G}=\Delta \mathrm{H}-\mathrm{T} \Delta \mathrm{S}
$$

\begin{tabular}{|c|c|c|c|c|c|}
\hline $\mathrm{pH}$ & $\begin{array}{c}\text { Incubation time } \\
\text { (min) }\end{array}$ & Temperature (K) & $\mathrm{K}_{\mathrm{sv}}\left(\mathrm{x} 10^{\wedge} 6\right) \mathrm{L} / \mathrm{mol}$ & $T_{0}(n s)$ & $k_{Q}\left(x 10^{\wedge} 15\right)$ L/mol.s \\
\hline \multirow[t]{2}{*}{7.4} & \multirow[t]{2}{*}{45} & 298 & 0.1654 & \multirow[t]{2}{*}{5} & 0.0331 \\
\hline & & 310 & 0.1631 & & 0.0326 \\
\hline
\end{tabular}

Table 1b: Stern-Volmer constants $\left(K_{\mathrm{sv}}\right)$ for Quercetin-Bovine Serum Albumin complex (incubation temperatures

\begin{tabular}{|c|c|c|c|c|c|}
\hline $\mathrm{pH}$ & $\begin{array}{c}\text { Incubation time } \\
\text { (min) }\end{array}$ & Temperature (K) & $K_{s v}\left(x 10^{\wedge} 6\right) L / m o l$ & $T_{0}(n s)$ & $k_{Q}\left(x 10^{\wedge} 15\right)$ L/mol.s \\
\hline \multirow[t]{2}{*}{7.4} & \multirow[t]{2}{*}{45} & 298 & 0.0752 & \multirow[t]{2}{*}{5} & 0.0150 \\
\hline & & 310 & 0.0739 & & 0.0148 \\
\hline
\end{tabular}
maintained at $298 \mathrm{~K}$ and $310 \mathrm{~K}$ respectively for $45 \mathrm{mins}$ for each sample).

Table 2a: The number of binding sites and binding constants for the LuteolinBovine Serum Albumin system at different temperatures.

\begin{tabular}{|c|c|c|c|c|c|}
\hline $\mathbf{p H}$ & $\begin{array}{c}\text { Incubation time } \\
(\mathbf{m i n})\end{array}$ & Temperature (K) & $\mathbf{K}(\mathbf{L} / \mathbf{m o l})$ & $\mathbf{n}$ & $\mathbf{R}^{2}$ \\
\hline 7.4 & 45 & 298 & $2.5421 \times 10^{\wedge} 6$ & 1.2785 & 0.9928 \\
\cline { 2 - 5 } & & 310 & $2.3174 \times 10^{\wedge} 6$ & 1.2695 & 0.9938 \\
\hline
\end{tabular}

\begin{tabular}{|} 
Table 2b: Binding constants and number of binding sites for the Quercetin- \\
Bovine Serum Albumin system at different temperatures. \\
\hline pH & $\begin{array}{c}\text { Incubation time } \\
\text { (min) }\end{array}$ & Temperature (K) & K (L/mol) & $\mathbf{n}$ & $\mathbf{R}^{2}$ \\
\hline 7.4 & 45 & 298 & $1.3568 \times 10^{\wedge} 6$ & 1.2799 & 0.9944 \\
\cline { 3 - 6 } & & 310 & $1.7128 \times 10^{\wedge} 5$ & 1.0648 & 0.9938 \\
\hline
\end{tabular}


The data obtained from the thermodynamic parameters of flavonoid-BSA systems displayed positive entropy change $\Delta \mathrm{S}_{0}$, which is indicative of the presence of hydrophobic interactions. This is because the orderly arrangement of water molecules around the flavonoid and the proteins configuration becoming random. A negative $\Delta \mathrm{H}_{0}$ value represents hydrogen bonding in the flavonoid-BSA system (Table $3 \mathrm{a}$ and Table $3 \mathrm{~b}){ }^{5}$ Hill's coefficient is a vital tool in analyzing the cooperativity (positive, negative and non-cooperative) of the binding of luteolin and quercetin respectively to BSA. ${ }^{10}$ The data below indicated a mostly non-cooperative nature of binding (under our defined experimental conditions) (Table 4).

The Hill's coefficient was calculated based on the following equation:

Where,

$$
\log \mathrm{Y} /(1-\mathrm{Y})=\log \mathrm{K}+\mathrm{nH} \log [\mathrm{L}] \quad-\text { Equation } 5
$$

$\mathrm{Y}=$ Fractional binding saturation

$\mathrm{K}=$ Binding constant

$\mathrm{nH}=$ Hill's coefficient

FTIR spectroscopy (Figures 5a, 5b and $6 \mathrm{a}$ and $6 \mathrm{~b}$ ) was performed to determine the alterations in the secondary structure of BSA following a separate binding experiment with Luteolin and Quercetin respectively. There were IR peaks observed in the pure BSA sample at around $3300 / \mathrm{cm}$ which suggests medium intensity Alkene/Aromatic $\mathrm{C}-\mathrm{H}$ stretching. The peak near $1635 / \mathrm{cm}$ is the amide $\mathrm{I}$ band. This band is due to the $\mathrm{C}=\mathrm{O}$ stretching vibrations of the peptide bond. The medium intensity C-N stretching was due to $1360 / \mathrm{cm}$ and $1215 / \mathrm{cm}$ respectively. Similarly, the peaks near $2980 / \mathrm{cm}$ is a result of a strong C-H stretching. The band at $1040 / \mathrm{cm}$ is due to strong C-O stretching while that at $870 / \mathrm{cm}$ is due to a strong $=\mathrm{C}-\mathrm{H}$ bending, following interactions of the flavonoids respectively with BSA. These results indicate that there might be conjugation between the flavonoid and BSA. Finally, in vitro molecular spectroscopy and thermodynamic data was corroborated through molecular docking studies. These docking studies were also carried out separately for luteolin and quercetin for both BSA and HSA.

Docking studies were performed on BSA and HSA (PDBID: 4F5S and $1 \mathrm{AO} 6$ respectively) to identify the possible binding site of Luteolin and Quercetin. According to the best energy ranked result of BSALuteolin docking $(-4.53 \mathrm{kcal} / \mathrm{mol})$, two hydrogen bonds were established between the $\mathrm{O} 5$ atom attached on the C12 of Luteolin and ARG144 residue of BSA as well as between $\mathrm{O} 5$ atom attached to the C12 of Luteolin and LEU115 residue of BSA (Figure 7). Similarly, the best energy ranked result of BSA-Quercetin docking (-3.16 $\mathrm{kcal} / \mathrm{mol}$ ) showed that the interaction between BSA QUE had five hydrogen bonds established between the O13 atom attached to the C9 of Quercetin and LEU 304 of BSA, between the O27 atom attached on the C10 of Quercetin and ARG336 of BSA, between the O27 atom attached on the C10 of Quercetin and LEU 301 of BSA, between the $\mathrm{O} 18$ atom attached on the C23 of Quercetin and GLU 299 of BSA and between the $\mathrm{O} 24$ atom attached on the C17 of Quercetin and GLU 299 of BSA (Figure 8). The best energy ranked

\begin{tabular}{|c|c|c|c|c|c|}
\hline \multicolumn{5}{|c|}{ Table 3a: Relative thermodynamic parameters of the Luteolin-Bovine Serum Albumin system. } \\
\hline $\mathbf{p H}$ & Incubation time $(\mathbf{m i n})$ & Temperature $(\mathbf{K})$ & $\boldsymbol{\Delta} \mathbf{H}^{\circ}(\mathbf{k J} / \mathbf{m o l})$ & $\boldsymbol{\Delta} \mathbf{G}^{\circ}(\mathbf{k J} / \mathbf{m o l})$ & $\boldsymbol{\Delta} \mathbf{S}^{\circ}(\mathbf{J} / \mathbf{m o l} . \mathbf{K})$ \\
\hline 7.4 & 45 & 298 & -0.0857 & -0.5286 & 1.4863 \\
\cline { 5 - 6 } & & 310 & & -0.5464 & \\
\hline
\end{tabular}

Table 3b: Relative thermodynamic parameters of Quercetin-Bovine Serum Albumin system.

\begin{tabular}{|c|c|c|c|c|c|}
\hline $\mathbf{p H}$ & Incubation time (min) & Temperature $(\mathbf{K})$ & $\boldsymbol{\Delta} \mathbf{H}^{\circ}(\mathbf{k J} / \mathbf{m o l})$ & $\boldsymbol{\Delta} \mathbf{G}^{\circ}(\mathbf{k J} / \mathbf{m o l})$ & $\boldsymbol{\Delta} \mathbf{S}^{\circ}(\mathbf{J} / \mathbf{m o l} . \mathbf{K})$ \\
\hline \multirow{2}{*}{7.4} & 45 & 298 & -1.9163 & -3.3265 & 4.7321 \\
\cline { 3 - 3 } & & 310 & & -3.3832 & \\
\hline
\end{tabular}

\begin{tabular}{|c|c|c|c|c|}
\hline \multicolumn{5}{|c|}{ Table 4: Hill's coefficients of Flavonoid-Bovine Serum Albumin systems. } \\
\hline SI.No. & $\begin{array}{c}\text { Molecules involved in the } \\
\text { interaction }\end{array}$ & Time (Min) & $\begin{array}{c}\text { Temperature } \\
\text { (Kelvin -K) }\end{array}$ & Hill's Coefficients \\
\hline 1a & BSA-Luteolin & 45 & $298 \mathrm{~K}$ & 0.9601 \\
\hline 1b & BSA-Luteolin & 45 & $310 \mathrm{~K}$ & 0.9978 \\
\hline 2a & BSA-Quercetin & $45 \mathrm{~min}$ & $298 \mathrm{~K}$ & 0.8792 \\
\hline 2b & BSA-Quercetin & $45 \mathrm{~min}$ & $301 \mathrm{~K}$ & 1.0994 \\
\hline
\end{tabular}




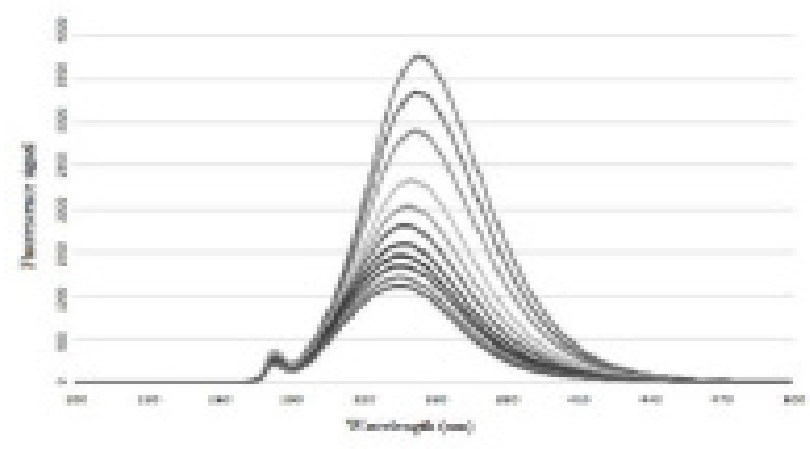

Figure 4: Fluorescence emission spectra of Bovine Serum Albumin complexed with quercetin ranging from $3.3 \mu \mathrm{M}$ to $33 \mu \mathrm{M}$ (slit width $5 \mathrm{~nm}$, excitation wavelength $280 \mathrm{~nm}$, temperature 25 degree $\mathrm{C}$, incubation time- $45 \mathrm{~min}, \mathrm{pH}$ 7.4).

The fluorescence intensity of Bovine Serum Albumin decreased, as the concentration of Quercetin became higher. A hypsochromic shift (Blue shift) is observed.

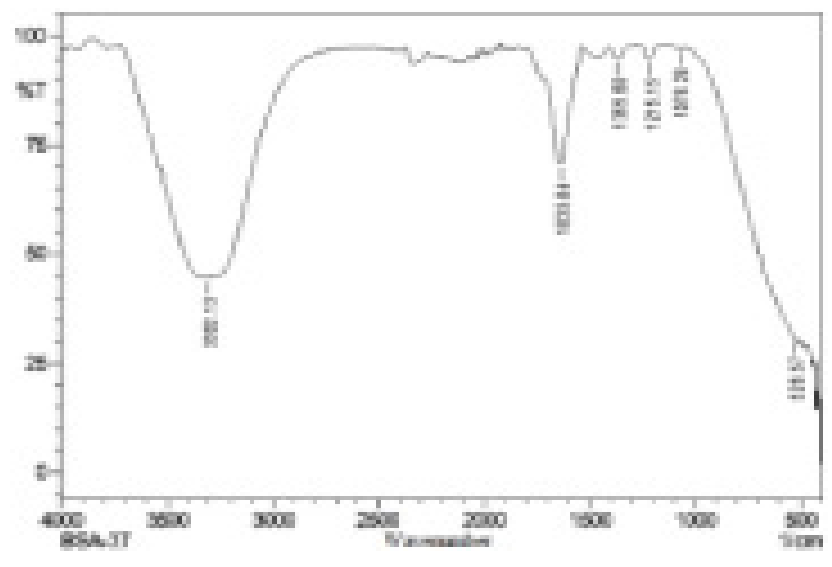

Figure 5a: Fourier Transform-Infra Red spectra for Quercetin-Bovine Serum Albumin interactions.

a) Bovine Serum Albumin at $310 \mathrm{~K}$.

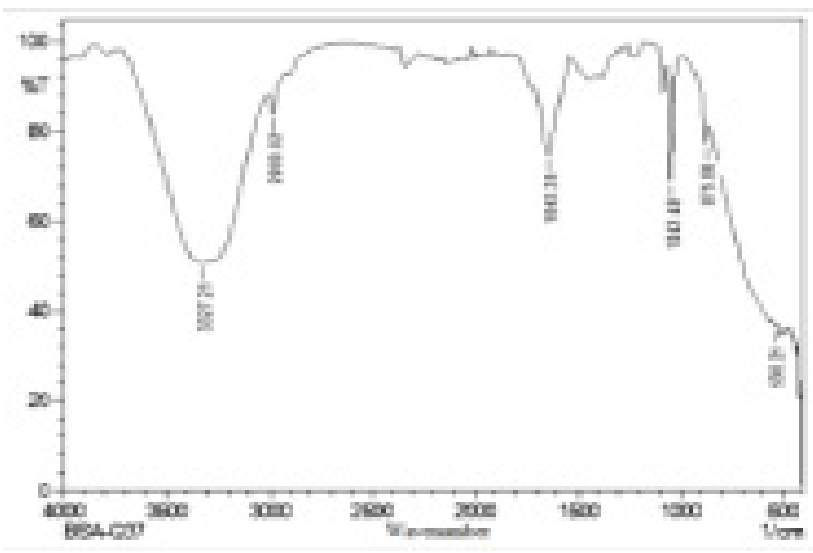

Figure 5b: Fourier Transform-Infra Red spectra for Quercetin-Bovine Serum Albumin interactions. b)Bovine Serum Albumin-Quercetin complex at $310 \mathrm{~K}$

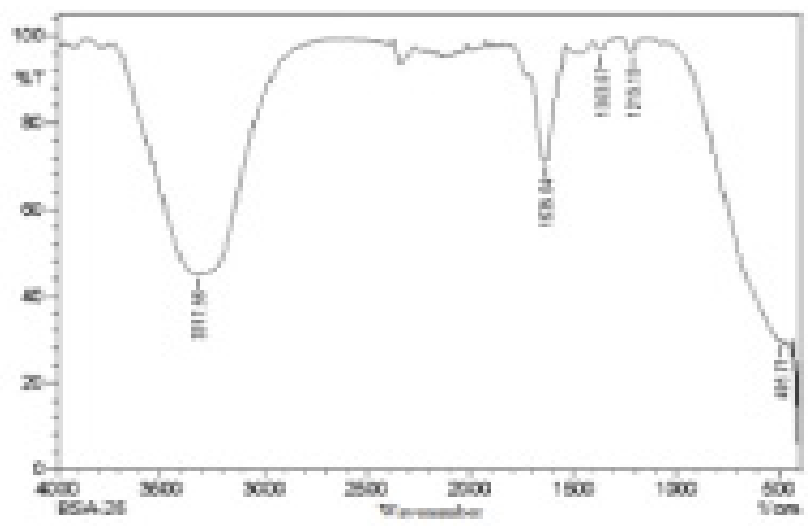

Figure 5c: Fourier Transform-Infra Red spectra for Quercetin-Bovine Serum Albumin interactions. c) Bovine Serum Albumin at $298 \mathrm{~K}$.

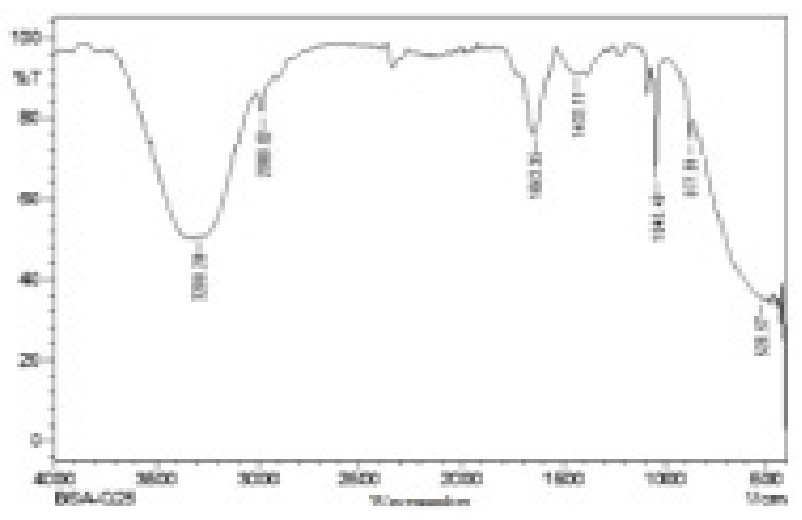

Figure 5d: Fourier Transform-Infra Red spectra for Quercetin-Bovine Serum Albumin interactions.

d) Bovine Serum Albumin-Quercetin complex at $298 \mathrm{~K}$.

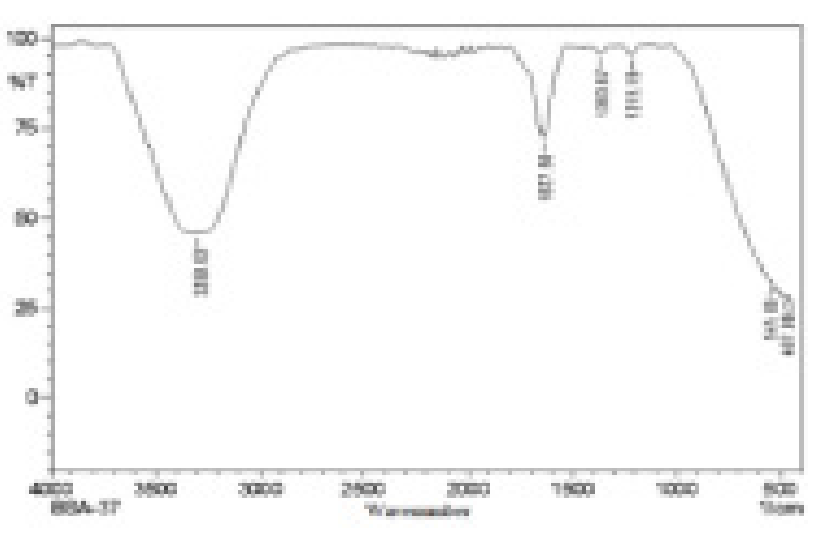

Figure 6a: Fourier Transform-Infra Red spectra for Luteolin-Bovine Serum Albumin interactions. a) Bovine Serum Albumin at $310 \mathrm{~K}$. 


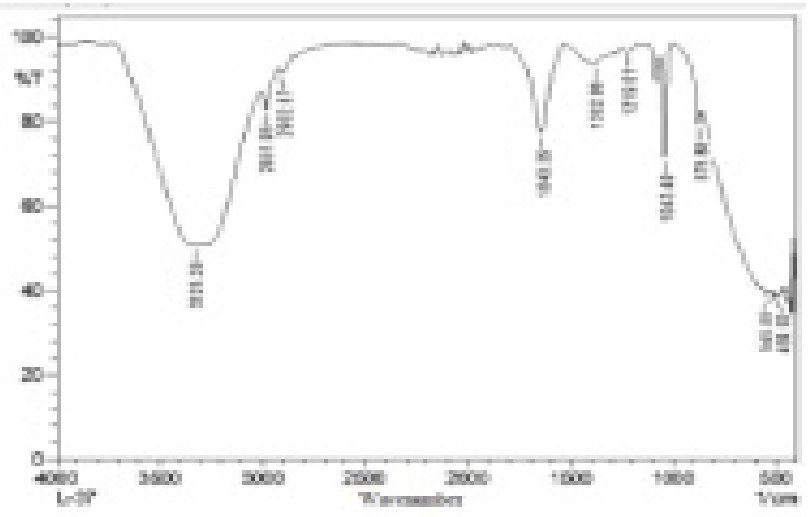

Figure 6b: Fourier Transform-Infra Red spectra for Luteolin-Bovine Serum Albumin interactions.

b) Bovine Serum Albumin-Luteolin complex at 310K.

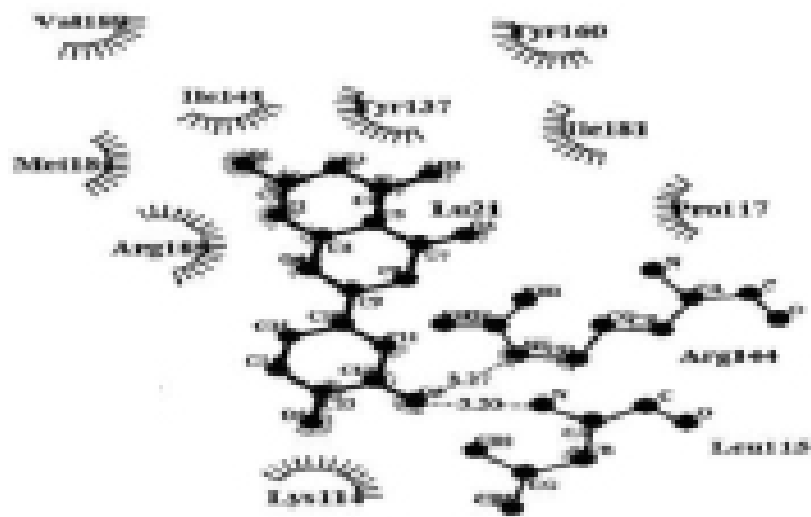

Figure 7: Hydrogen bonds were established between the 05 atom attached on the C12 of Luteolin and ARG144 residue of Bovine Serum Albumin as well as between 05 atom attached on the $\mathrm{C} 12$ of Luteolin and LEU115 residue of Bovine Serum Albumin.

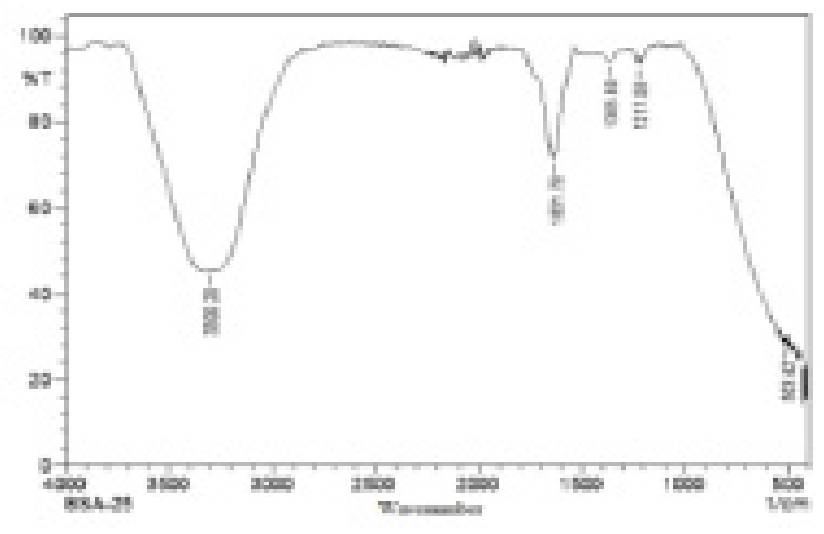

Figure 6c: Fourier Transform-Infra Red spectra for Luteolin-Bovine Serum Albumin interactions. c) Bovine Serum Albumin at $298 \mathrm{~K}$.

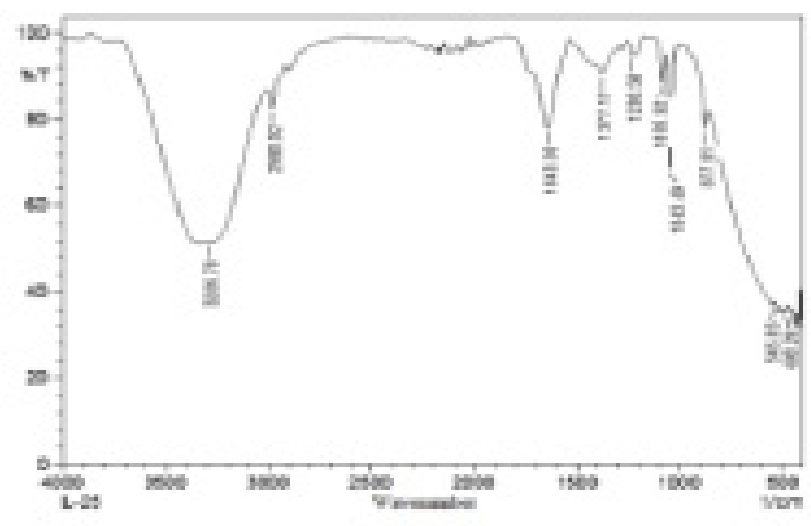

Figure 6d: Fourier Transform-Infra Red spectra for Luteolin-Bovine Serum Albumin interactions. d) Bovine Serum Albumin-Luteolin complex at $298 \mathrm{~K}$. result of HSA-Luteolin docking $(-4.92 \mathrm{kcal} / \mathrm{mol})$, three hydrogen bonds were established between the $\mathrm{O} 1$ atom attached to the $\mathrm{C} 2$ of Luteolin and LEU 115 residue of HSA as well as between O5 atom attached on the C12 of Luteolin and TYR 161 residue of HSA and between O5 atom attached on the C12 of Luteolin and PHE 134 residue of HSA (Figure 9). Similarly, the best energy ranked result of HSA-Quercetin docking $(-5.90 \mathrm{kcal} / \mathrm{mol})$ showed that the interaction between BSA-QUE had three hydrogen bonds established between the O29 atom attached to the C6 of Quercetin and LEU 182 of HSA, between the $\mathrm{O} 12$ atom attached to the $\mathrm{C} 4$ of Quercetin and TYR 161 of HSA and between the O24 atom attached on the C17 of Quercetin and PHE 134 of HSA (Figure 10). Docking studies further confirmed the presence of hydrophobic interactions as well as hydrogen bonds, (Table 5) thereby validating the interactions demonstrated by classical and widely accepted spectroscopic methods.

\section{DISCUSSION}

Binding studies of luteolin and quercetin (dietary ingredients with cancer chemo-preventive potential). ${ }^{11}$ with model proteins (like BSA) are important in terms of evaluating the nature and site of binding with ramifications for PK/PD parameters in drug development and/ or drug refinement. Further, experiments with model chemicals can also aid in improving our mechanistic understanding of the conformational changes in BSA, apart from providing insights into ligand-specific allostery and/or cooperativity-based interactions. Also, this design of ours provides a basis for extending this study to the use of luteolin or quercetin (chemicals with fluo- 


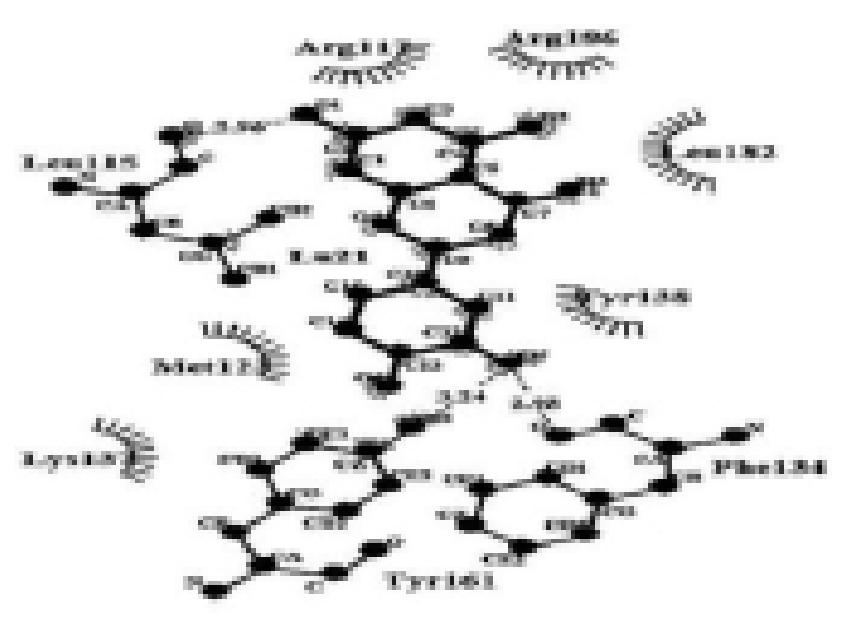

Figure 8: Hydrogen bonds established between the 013 atom attached on the C9 of Quercetin and LEU 304 of Bovine Serum Albumin, between the $\mathrm{O} 27$ atom attached on the $\mathrm{C} 10$ of Quercetin and ARG336 of Bovine Serum Albumin, between the 027 atom attached on the C10 of Quercetin and LEU

301 of Bovine Serum Albumin, between the 018 atom attached on the C23 of Quercetin and GLU 299 of Bovine Serum Albumin and between the $\mathrm{O24}$ atom attached on the C17 of Quercetin and GLU 299 of Bovine Serum Albumin.

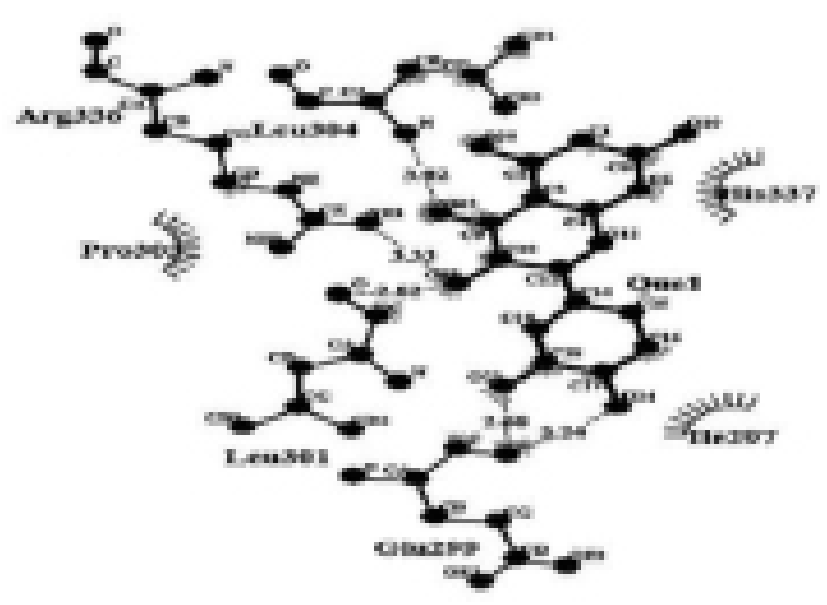

Figure 9: Hydrogen bonds were established between the 01 atom attached on the C2 of Luteolin and LEU 115 residue of Human Serum Albumin as well as between 05 atom attached on the C12 of Luteolin and TYR 161 residue of HSA and between $\mathrm{O} 5$ atom attached on the $\mathrm{C} 12$ of Luteolin and PHE 134 residue of Human Serum Albumin.

rescence quenching properties) as experimental probes to evaluate the fluorescence behaviour of other receptors that have fluorophores in them. These experiments will also validate the utility of this in vitro in silico experimental design to screen existing and novel drugs/ ligands with existing/novel receptors for their binding potential. The UV-visible spectra validated the quality of the BSA, luteolin and quercetin used in our experiments (data not shown). A characteristic hypsochromic shift was observed for both Luteolin $(3 \mathrm{~nm})$ and

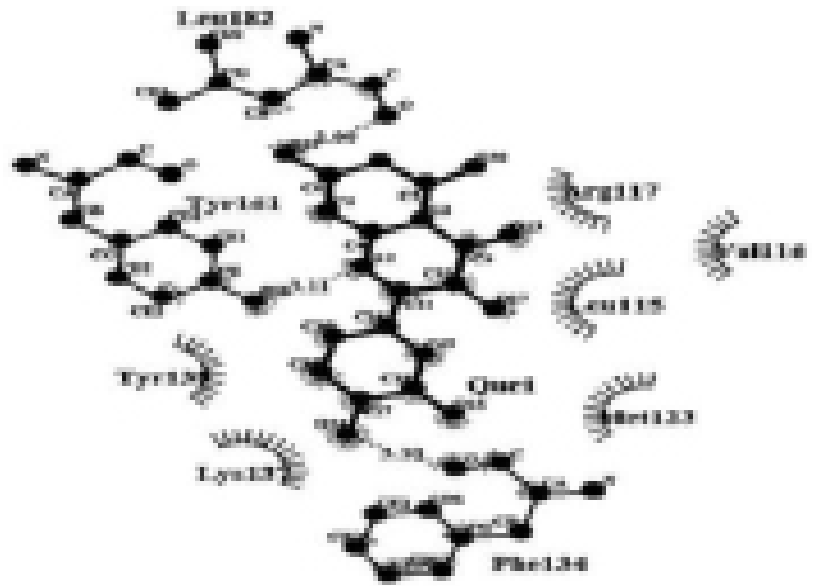

Figure 10: Hydrogen bonds established between the 029 atom attached on the C6 of Quercetin and LEU 182 of Human Serum Albumin, between the $\mathbf{0 1 2}$ atom attached on the $\mathrm{C} 4$ of Quercetin and TYR 161 of Human Serum Albumin and between the $\mathrm{O} 24$ atom attached on the $\mathrm{C} 17$ of Quercetin and PHE 134 of Human Serum Albumin.

Quercetin (4.1 nm) following binding to BSA (Figures 1 and 2). This blue shift can be attributed to a conformational change or a transitions in the protein resulting in the tryptophan chromophore being placed in a less polar, more hydrophobic microenvironment. ${ }^{12}$ Other reasons could be alterations in the associations of the subunits, denaturation of the protein or may be dependent on substrate binding-mediated events. ${ }^{13}$

To the best of our knowledge, there are no reports wherein a systematic comparison has been made with respect to the reproducible, luteolin versus quercetinmediated UV-visible hypsochromic shifts, using a particular range of concentrations $(3.33-33 \mu \mathrm{M}$ of luteolin versus quercetin) with a constant concentration of BSA $\left(1.51 \times 10^{-5} \mathrm{M}=15.1 \mu \mathrm{M}\right)$. Further, our report has specifically documented a hitherto unreported UV-visible spectroscopy-based 3 and $4.1 \mathrm{~nm}$ shifts for luteolin and quercetin respectively. Ni et al., $2009^{7}$ adopted a different experimental design in terms of keeping the concentrations of both quercetin $(16.5 \mu \mathrm{M})$ and BSA $(15.0 \mu \mathrm{M})$ constant. They have reported a qualitatively similar hypsochromic shift with respect to BSA. Zhang et al., $2011^{13}$ have also reported a similar qualitative blue shift with respect to quercetin $(0-10 \mu \mathrm{M})$ only. Shifts pertaining to luteolin were not shown. Further, they used a different concentration of BSA $(6 \mu \mathrm{M})$. In the case of Tang et al., $2013,{ }^{14}$ there was a red shift in the case of the BSA peak $(203 \mathrm{~nm})$ to $205 \mathrm{~nm}$ for luteolin $(0-18 \mu \mathrm{M})$ only. Also, they have used a different concentration of BSA $(0.5 \mu \mathrm{M})$. We have demonstrated a decrease in fluorescence intensity with increasing concentration of luteolin and quercetin (separate experiments) (Figure 3 and 4). The 
Table 5: Docking Results for Luteolin and Quercetin (Binding Energy, number of hydrogen bonds and the nature of binding)

\begin{tabular}{|c|c|c|c|}
\hline Compounds & $\begin{array}{l}\text { Best energy } \\
\text { rank (kcal/mol) }\end{array}$ & $\begin{array}{c}\text { Number of Hydrogen } \\
\text { bonds }\end{array}$ & Hydrogen bonds between \\
\hline \multirow[t]{2}{*}{ BSA-Luteolin } & \multirow[t]{2}{*}{-4.53} & \multirow[t]{2}{*}{2} & O5 atom attached on the C12 of Luteolin and ARG144 residue of BSA \\
\hline & & & O5 atom attached to the C12 of Luteolin and LEU115 residue of BSA \\
\hline \multirow[t]{5}{*}{ BSA-Quercetin } & \multirow[t]{5}{*}{-3.16} & \multirow[t]{5}{*}{5} & O13 atom attached to the C9 of Quercetin and LEU 304 of BSA \\
\hline & & & O27 atom attached on the C10 of Quercetin and ARG336 of BSA \\
\hline & & & O27 atom attached on the C10 of Quercetin and LEU 301 of BSA \\
\hline & & & O18 atom attached on the C23 of Quercetin and GLU 299 of BSA \\
\hline & & & O24 atom attached on the C17 of Quercetin and GLU 299 of BSA \\
\hline \multirow[t]{3}{*}{ HSA-Luteolin } & \multirow[t]{3}{*}{-4.92} & \multirow[t]{3}{*}{3} & O1 atom attached to the C2 of Luteolin and LEU 115 residue of HSA \\
\hline & & & O5 atom attached on the C12 of Luteolin and TYR 161 residue of HSA \\
\hline & & & O5 atom attached on the $\mathrm{C} 12$ of Luteolin and PHE 134 residue of HSA \\
\hline \multirow[t]{3}{*}{ HSA-Quercetin } & \multirow[t]{3}{*}{-5.90} & \multirow[t]{3}{*}{3} & O29 atom attached to the C6 of Quercetin and LEU 182 of HSA \\
\hline & & & O12 atom attached to the C4 of Quercetin and TYR 161 of HSA \\
\hline & & & O24 atom attached on the C17 of Quercetin and PHE 134 of HSA \\
\hline
\end{tabular}

hypsochromic shift was also replicated following fluorescence spectroscopy measurements. ${ }^{15}$

Other reports using a different concentration/range of concentration of luteolin or quercetin and BSA) have documented similar shifts. Specifically, luteolin (0.75-7.5 $\mu \mathrm{M})$-induced blue shift ${ }^{5}$ and a $5 \mathrm{~nm}$ shift for the same compound $(0-10 \mu \mathrm{M})$ have been reported. ${ }^{14}$ In the case of quercetin $(6 \mu \mathrm{M})$, a slight blue shift of $2-3 \mathrm{~nm}$ has been demonstrated. ${ }^{15}$ Similar shift $(\sim 5 \mathrm{~nm})$ has been reported by other researchers, despite differences in the absolute emission wavelength maxima. ${ }^{16}$

Fluorescence spectroscopy results seem to point towards a static quenching mechanism under our defined experimental conditions (Table $1 \mathrm{a}$ and 1b)., $5,7,8,10,13,14,17$ There have been very few reports wherein a comparison has been made between luteolin and quercetin with respect to fluorescence spectroscopy data. ${ }^{13,17}$ Unlike the experimental flow in these reports, we have adopted an experimental design wherein thermodynamic and docking data have also been incorporated (please see below). Further, the possibility of dynamic quenching also playing a role in this process cannot be ruled out since measurements taken following incubation for a shorter duration may have revealed the relative contribution of dynamic quenching ${ }^{2,14}$ in this process. Hence, caution should also be exercised in terms of our interpretation of the mechanisms of the fluorescence quenching phenomena (relative contribution by dynamic quenching phenomena). The binding constant values, being in excess of $105 \mathrm{~L} / \mathrm{mol}$ provided evidence of a strong binding force which is consistent with the data reported by others. ${ }^{5,8,10,13,14,16}$ The number of binding site being 1 for both luteolin and quercetin is in keeping with the results obtained by Zhang et al. 2011 (Table 2a and Table 2b). ${ }^{13}$ Despite other papers documenting a number higher than 1, raising the possibility of the existence of more than one binding site, ${ }^{5,8,14,16}$ definitive evidence regarding this is lacking. The positive entropy change $\Delta \mathrm{S}_{0}$ is indicative of the presence of hydrophobic interactions. This is because of the orderly arrangement of water molecules around the flavonoid and the configuration of the protein is random. A negative $\Delta \mathrm{H}_{0}$ value represents hydrogen bonding in the flavonoid-BSA system. Last but not least, a negative $\Delta \mathrm{H}_{0}$ and a positive $\Delta \mathrm{S}_{0}$, shown by us, provides evidence for the possibility of electrostatic interactions (Table $3 \mathrm{a}$ and Table $3 \mathrm{~b}$ ) Despite variations in the magnitude of the respective thermodynamic values, a striking qualitative correlation was observed ${ }^{5,8,14,16}$ with respect to all the thermodynamic variables. Under our defined experimental conditions, the Hill's coefficient data of ours pointed towards a hitherto unreported non-cooperative nature of the binding (Table 4). The FT-IR data has demonstrated certain shifts in the wavenumbers providing an evidence of the possible interactions of luteolin and quercetin with BSA (under our defined experimental conditions). Specifically, the broad band in the region between $4000-2400 \mathrm{~cm}-1$ observed for luteolin and is due to the H-bonding between the $\mathrm{C}=\mathrm{O}$ and $5-\mathrm{OH}$ group. ${ }^{15}$ A similar broad band in the same region was also demonstrated by us for quercetin and is consistent with the interactions between the $\mathrm{C}=\mathrm{O}$ and the $5-\mathrm{OH}$ group. The $\mathrm{C}=\mathrm{O}$ stretching band at $1650 \mathrm{~cm}-1$ is considered to be the amide I band ${ }^{15}$ and may be due to the presence 
of beta sheets. ${ }^{16}$ The absence of amide II band results may be due to alterations in the secondary structure due to the binding of luteolin and quercetin (Figure 5 and 6 ). However, this aspect should be verified before any firm conclusions can be drawn. To the best of our knowledge, this report of ours is the first of its kind to include FT-IR data in our in vitro in silico experimental flow for comparing luteolin and quercetin. The preponderance of hydrogen bonds and hydrophobic residues (in our LIGPLOT results) provide corroborative evidence of molecular spectroscopy and thermodynamic data. (Figure 7, second 8, 9 and 10). The docking results seem to be conflicting with regards to the binding affinity of luteolin and quercetin with BSA and HSA respectively. Specifically, luteolin has a higher binding affinity to BSA in comparison with the results obtained for quercetin. Conversely, quercetin has a higher binding affinity for HSA in comparison with that of luteolin. These contradictory results are also mirrored in the experimental findings in terms of quenching. It has been demonstrated that quecetin is better than luteolin ${ }^{16}$ while another research group has reported that luteolinmediated decrease in BSA fluorescence is higher than that of quercetin. ${ }^{15}$

The corroborative data obtained using different methodologies increase the strength of evidence about the general nature of interactions in the visualized docked complexes (LIGPLOT data). However, variability in the amino acids involved ${ }^{18}$ may be attributable to the inherent random nature of the selection of the different ligand poses by AUTODOCK 4.2. Our unique, combinatorial, in vitro/in silico experimental flow involved the use of multiple spectroscopy-based tools; including the hitherto unreported FT-IR data as well as the determination of Hill's coefficient; and the thermodynamic values. This approach, when thoroughly validated, can be used to screen for the nature and site of interactions of novel receptors and/or novel ligands. Last but not least, this flow can be a teaching tool for demonstrating interactions between a model, hydrophobic protein (BSA) and natural ethno-based drugs (for e.g., luteolin and quercetin).

\section{CONCLUSION}

This in silico in vitro combinatorial experimental flow used in our experiments is the first of its kind (this particular combination of analytical and in silico tools) in terms of comprehensively analyzing the binding characteristics of luteolin in comparison with that of quercetin. The same flow can be used as a teaching tool and can be extended for other ligands and receptors as well. However, we need to repeat the experiments with HSA using our in vitro in silico experimental flow. This approach is necessitated to evaluate the predictability of our binding protocols and in silico analysis for human hydrophobic proteins (other than BSA and HSA -existing and novel) as well as other ligands (existing and novel -not necessarily restricted to those in the flavonoid class).

\section{ACKNOWLEDGEMENT}

The authors thank VIT for their constant encouragement and support as well as for their unlimited WIFI connectivity. The first author of this paper thanks DST (SERB) for funding a project on drug delivery systems involving Luteolin ((SB/SO/HS-157 (2013)). Last but not least, the lead author also thanks the research scholars and other students for their support.

\section{CONFLCIT OF INTEREST}

The authors declare no conflict of interest.

\section{ABBREVIATIONS USED}

BSA: Bovine Serum Albumin, $\mathbf{\Delta} \mathbf{S 0}$ : Change is entropy, $\Delta \mathbf{H 0 :}$ Change in enthalpy, FT-IR: Fourier Transform Infrared Spectroscopy, HSA: Human Serum Albumin, Trp: Tryptophan, Arg: Arginine, Leu: Leucine, Glu: Glutamic acid, Tyr: Tyrosine, Phe: Phenylalanine, QUE: Quercetin, PBS: Phosphate Buffer Saline, $\lambda$ max : Lambda Max, PDB: Protein Data Bank, kQ: Quenching rate constant, KSV: Stern-Volmer Quenching constant, F: Fluorescence intensity with quencher, F0: Fluorescence intensity without quencher, [Q]: Quencher concentration, $\tau 0$ : Average fluorescence lifetime of the biomolecule without the quencher, $\Delta \mathbf{G} \mathbf{0}$ : Gibbs free energy change, $\mathbf{R}$ : Gas constant, n: Number of binding sites, Y: Fractional binding saturation, $\mathbf{K}$ : Binding constant, $\mathbf{n H}$ : Hill's coefficient.

\section{REFERENCES}

1. Tayeh N, Rungassamy T. Albani JR. Fluorescence spectral resolution of tryptophan residues in bovine and human serum albumins. Journal of Pharmaceutical and Biomedical Analysis. 2009;50(2):107-16.

2. Papadopoulou A, Green RJ, Frazier RA. Interaction of flavonoids with bovine serum albumin: A fluorescence quenching study. Journal of Agricultural and Food Chemistry. 2005;53(1):158-63.

3. Havsteen $\mathrm{BH}$. The biochemistry and medical significance of the flavonoids Pharmacology and Therapeutics. 2002;96(2-3):67-202.

4. Poór M, Li Y, Matisz G, Kiss L, Kunsági-Máté S, Köszegi T. Quantitation of species differences in albumin-ligand interactions for bovine, human and rat serum albumins using fluorescence spectroscopy: A test case with some Sudlow's site I ligands Journal of Luminescence. 2014;145: 767-73. 
5. Yang $\mathrm{Y}, \mathrm{Hu} \mathrm{Q}$, Fan $\mathrm{Y}$, Shen $\mathrm{H}$. Study on the binding of luteolin to bovine serum albumin. Spectrochim. Acta - Part A Molecular and Biomolecular Spectroscopy. 2008;69(2):432-6.

6. Liu B, Yang C, Yan X, Wang J, Lv Y. Study on the conjugation mechanism of colistin sulfate with bovine serum albumin and effect of the metal ions on the reaction. Journal of Luminescence. 2012;132(5):1133-8.

7. $\mathrm{Ni} \mathrm{Y}$, Zhang $\mathrm{X}$, Kokot $\mathrm{S}$. Spectrometric and voltammetric studies of the interaction between quercetin and bovine serum albumin using warfarin as site marker with the aid of chemometrics. Spectrochim. Acta - Part A Molecular and Biomolecular Spectroscopy. 2009;71(5):1865-72.

8. Fu L, Sun Y, Ding L, Wang Y, Gao Z, Wu Z, Wang S, Li W, Bi Y. Mechanism evaluation of the interactions between flavonoids and bovine serum albumin based on multi-spectroscopy, molecular docking and Q-TOF HR-MS analyses Food Chemistry. 2016;203:150-7.

9. Hossain M, Khan AY, Kumar GS. Interaction of the anticancer plant alkaloid sanguinarine with bovine serum albumin PLoS One. 2011;6(4):e18333.

10. Skrt M, Benedik E, Podlipnik Č, Ulrih NP. Interactions of different polyphenols with bovine serum albumin using fluorescence quenching and molecular docking Food Chemistry. 2012;135(4):2418-24.

11. Bi S, Yan L, Pang B, Wang Y. Investigation of three flavonoids binding to bovine serum albumin using molecular fluorescence technique Journal of Luminescence. 2012;132(1):132-40.

12. Garzón A, Bravo I, Carrión-Jiménez MR, Rubio-Moraga Á, Albaladejo J. Spectroscopic study on binding of gentisic acid to bovine serum albumin Spectrochim Acta - Part A Molecular and Biomolecular Spectroscopy. 2015;150:26-33.
13. Zhang $\mathrm{Y}$, Shi S, Sun $\mathrm{X}$, Xiong $\mathrm{X}$, Peng $\mathrm{M}$. The effect of $\mathrm{Cu} 2+$ on interaction between flavonoids with different $\mathrm{C}$-ring substituents and bovine serum albumin: Structure - affi nity relationship aspect Journal of Inorganic Biochemistry. 2011;105(12):1529-37.

14. Tang L, Jia WA. comparison study on the binding of hesperetin and luteolin to bovine serum albumin by spectroscopy Spectrochim Acta - Part A Molecular and Biomolecular Spectroscopy. 2013;103:114-9.

15. Shi S, Zhang $Y$, Xiong $X$, Huang $K$, Chen $X$, Peng $M$. The influence of flavonoids on the binding of pantoprazole to bovine serum albumin by spectroscopic methods: With the viewpoint of food/drug interference Food Chemistry. 2012;135(3):1083-90.

16. Naso LG, Lezama L, Valcarcel M, Salado C, Villacé P, Kortazar D, Ferrer EG, Williams PA. Bovine serum albumin binding, antioxidant and anticancer properties of an oxidovanadium(IV) complex with luteolin Journal of Inorganic Biochemistry. 2016;157:80-93.

17. Roy D, Dutta S, Maity SS, Ghosh S, Roy AS, Ghosh KS, Dasgupta S. Spectroscopic and docking studies of the binding of two stereoisomeric antioxidant catechins to serum albumins Jounal of Luminescence. 2012;132(6):1364-75.

18. Johari A, Moosavi-Movahedi AA, Amanlou M. Computational investigation of inhibitory mechanism of flavonoids as bovine serum albumin anti-glycation agents Daru. 2014;22(1):79.

\section{PICTORIAL ABSTRACT}

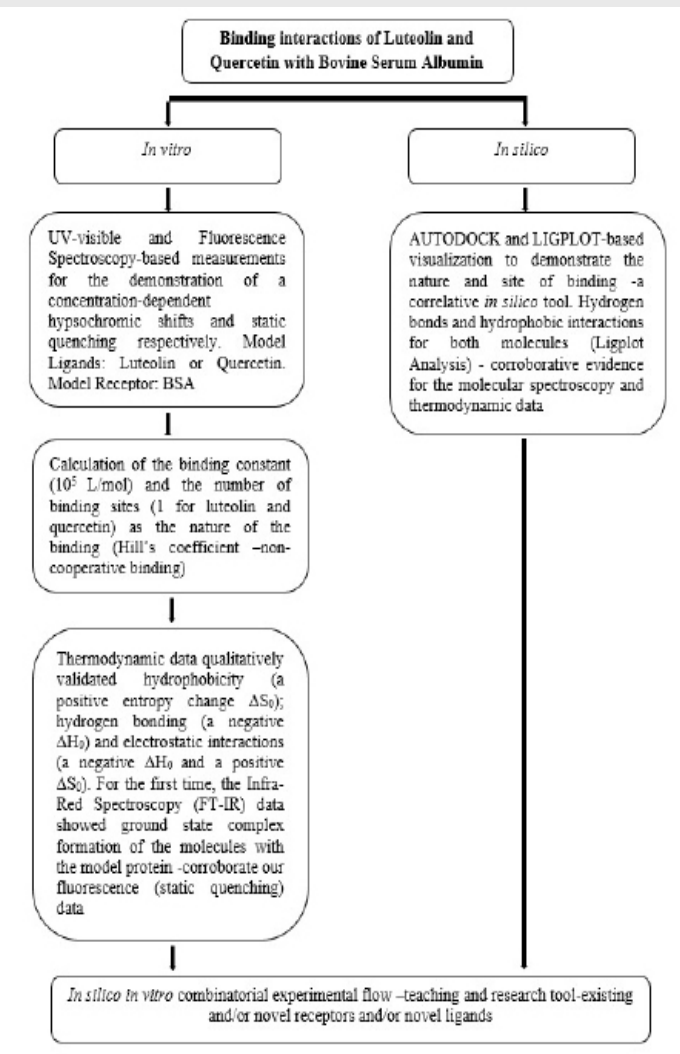

\section{SUMMARY}

- Systematic comparison showed reproducible UV-Vis spectroscopy-based hypsochromic shifts for both Luteolin and Quercetin-mediated interactions with BSA.

- Demonstration of a reproducible, static, Luteolin/Quercetinmediated quenching mediated interactions with BSA.

- The binding constant values, being in excess of $105 \mathrm{~L} / \mathrm{mol}$ provided evidence of a strong binding force.

- The number of binding site was found to be 1 for both luteolin and quercetin under our defined experimental conditions.

- Despite variations in the magnitude of the respective thermodynamic values, a striking qualitative correlation was observed with respect to all the thermodynamic variables.

- $\quad$ FT-IR spectra- possible presence of hydrogen bonding and beta sheets.

- $\quad$ LIGPLOT analysis -Preponderance of hydrogen bonds and hydrophobic interactions -corroborative evidence of molecular spectroscopy and thermodynamic data.

- hitherto unreported in silico in vitro combinatorial experimental flow - can be used as a teaching and research tool for novel receptors and/or novel ligands. 


\section{About Authors}

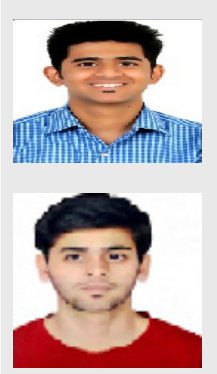

Hiray K.S: He has finished his Bachelor of Technology in Biotechnology from VIT Vellore. He is currently pursuing his Master of Technology by Research and is working on the effects of drugs (purified natural compounds) on cancer cells and formulation of an effective nano-derived drug delivery system.

Vyas S: He has completed his Bachelor of Technology in Biotechnology from VIT Vellore. He is currently employed at GeNext Genomics, a life science research firm. His role includes development of immune-assays as well as handling, screening and selection of high-affinity monoclonal antibodies.

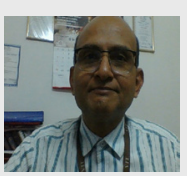

P.K.Suresh: Professor in the Department of Biomedical Sciences, School of Biosciences \& Technology. He has approximately 18 years of teaching, research and administrative experience (post-Ph.D.). He received his second masters \& Ph.D. in SIUE, IL, USA and the University of Cincinnati, Ohio, USA respectively. He was a Post-doctoral fellow at the University of Texas at Austin, TX, USA as well as Rutgers University, Piscataway, USA. P.K.Suresh has authored/co-authored over 40 publications in SCOPUS indexed journals. He has mentored students at several levels including those pursuing their doctoral degree. Apart from in silico in vitro Chemical Biology, he is also involved in drug development and delivery systems.

Cite this article: Suresh PK, Hiray KS, Vyas S. A Correlative Multi-Spectroscopy and Docking Study for the Modeling of Drug (Luteolin and Quercetin) Binding to Bovine Serum Albumin- A Tool for the Determination of Binding Characteristics to Receptor Proteins. Indian J of Pharmaceutical Education and Research. 2018;5(3):492504. 